\title{
Analysis of Human Tissue Densities: A new approach to extract features from medical images
}

\author{
Pedro P. Rebouças Filho ${ }^{a}$, Elizângela de S. Rebouças ${ }^{a}$, Leandro B. Marinho ${ }^{a}$, Róger M. Sarmento $^{\mathrm{a}}$, João Manuel R. S. Tavares ${ }^{\mathrm{b}}$, \\ Victor Hugo C. de Albuquerque ${ }^{\mathrm{c}, * *}$ \\ ${ }^{a}$ Instituto Federal de Educação, Ciência e Tecnologia do Ceará, Brazil \\ ${ }^{b}$ Instituto de Ciência e Inovação em Engenharia Mecânica e Engenharia Industrial, Departamento de Engenharia Mecânica, Faculdade de Engenharia, \\ Universidade do Porto, Porto, Portugal \\ ${ }^{c}$ Programa de Pós-Graduação em Informática Aplicada, Laboratrio de Bioinformtica, Universidade de Fortaleza, Fortaleza, Ceará, Brazil
}

\begin{abstract}
Identification of diseases based on processing and analysis of medical images is of great importance for medical doctors to assist them in their decision making. In this work, a new feature extraction method based on human tissue density patterns, named Analysis of Human Tissue Densities (AHTD) is presented. The proposed method uses radiological densities of human tissues in Hounsfield Units to tackle the extraction of suitable features from medical images. This new method was compared against: the Gray Level Co-occurrence Matrix, Hu's moments, Statistical moments, Zernike's moments, Elliptic Fourier features, Tamura's features and the Statistical Co-occurrence Matrix. Four machine learning classifiers were applied to each feature extractor for two CT image datasets:, one to classify lung disease in CT images of the thorax and the other to classify stroke in CT images of the brain. The attributes were extracted from the lung images in 5.2 milliseconds and obtained an accuracy of $99.01 \%$ for the detection and classification of lung diseases, while the attributes from the brain images were extracted in 3.8 milliseconds and obtained an accuracy of $98.81 \%$ for the detection and classification of stroke. These results show that the proposed method can be used to classify diseases in medical images, and can be used in real-time applications due to its fast extraction time of suitable attributes.
\end{abstract}

(c) 2017 Elsevier Ltd. All rights reserved.

\section{Introduction}

Identification of diseases using techniques of processing and analysis of medical images is nowadays of great importance to assist medical doctors in making accurate diagnoses. Consequently, various studies aiming to extract representative information from medical images in order to identify diseases have been developed. In [1], structural imaging biomarkers were proposed to predict the progression of Alzheimer's disease; in [2], textural features are extracted from multimodal MRI to compute a Prognostic value for glioblastoma; in [3], 3D lung fissures are identified in Computed Tomography (CT)

\footnotetext{
${ }^{* *}$ Corresponding author:

e-mail: victor albuquerque@unif or . br (Victor Hugo C. de Albuquerque)
}

images based on texture; in [4], a generalized framework is proposed for medical image classification; and in [5], a Spatial Interdependence Matrix (SIM) is applied to classify pulmonary diseases in CT images.

Computed Tomography (CT) was developed by the engineer Godfrey N. Hounsfield and the physicist Allan M. Comark in 1972. The two scientists were awarded the Nobel Prize for Medicine in 1979 [6]. Hounsfield and Cormack proved that mathematical formulae could be used in the production of images with sufficient detail to aid analyses of the human body. By quantifying an X-ray beam transmitted through the human body, information concerning the body tissues is obtained, thus creating CT [7]. Each slice of the CT aims to determine the composition of a single dry body and is formed by a set of pixels. The amount and thickness of the cuts are directly related to the pixel sizes, which influence the quality of the final image. Finer cuts produce sharper images. 
CT images have a great variety of shades of gray, which obey a scale, according to the attenuation value of each tissue of the human body. In homage to the inventor of the CT, this scale is called the Hounsfield scale and presents values from 1000 to -1000 and is expressed in Hounsfield Units (UH), where 0 is the attenuation value adopted for water and -1000 the attenuation value adopted for air [8].

The extraction of relevant image information is characterized by the computation of attributes from the input image that represent the structures of interest [9]. These attributes are commonly used as input data to a classifier which automatically identifies the structures or related characteristics, like shape or condition.

Statistical moments (SM) are computed using the distribution of the gray levels of an input image, and are therefore usually computed from the image histogram. These characteristics provide a statistical description of the relationship between the different image gray levels [9]. According to [10], central moments are very important in pattern recognition. These moments are obtained from the spatial moments of a structure by referring the spatial moments relative to the centroid of the structure. The main advantage of central moments is their invariance to translations, and can therefore, in many cases, be used to describe the shape of a structure [11].

$\mathrm{Hu}$ [12] proposed a theory of two-dimensional moment invariants for planar geometric figures. The principle of $\mathrm{Hu}$ 's moments (HM), also known as Invariant Moments, is to allow the recognition of structures independently of their position, size and orientation. Despite their invariant properties, HM are highly dependent on the spatial resolution of the image [13]. However, many authors have applied HM as global or local feature descriptors in pattern recognition problems [14].These moments are often used to recognize structures in general, because of their ability to describe the shape of structures eectively [15].

Haralick [16] proposed a methodology to describe textures based on second-order statistics, where features are derived from co-occurrence matrices, which are built by counting the dierent combinations of gray levels of an image according to certain directions [16]. Gray Level Co-occurrence Matrices (GLCMs) are the basis of various statistical measures known as Haralick's descriptors. This technique is widely used to characterize images in many dierent applications of pattern recognition $[17,18]$. Texture based analysis has also been widely used in dierent image based applications, such as medical image analysis [19], visual interpretation of remote sensing images [20] and image search [21].

In [22], a new set of rotation invariant features was presented. These features are the magnitudes of a set of orthogonal complex moments of the image known as Zernike moments (ZM). Scale and translation invariance are obtained by first normalizing the image with respect to these parameters using its regular geometrical moments.

Kuhl et al. [23] presented a procedure to obtain the Fourier coecients of a chain-encoded contour. The advantage of the Elliptic Fourier features (EFF) are that they do not require integration or the use of fast Fourier transform techniques, and that bounds on the accuracy of the image contour reconstruction are easy to especify.

Tamura et al. [24] developed textural features approximating visual perception on the basis of psychological experiments. The authors approximated, in computational form, six basic textural features, namely, coarseness, contrast, directionality, line-likeness, regularity, and roughness. In the most cases, only the first three of Tamura's features (TF) are used for Contentbased image retrieval. These features capture the high-level perceptual attributes of a texture effectively and are useful for image browsing.

Ramalho et al. [25] presented a rotation-invariant feature extraction methodology based on a structural approach using cooccurrence statistics (SCM). The results show that this method provides an ecient and fast way to analyze digital images.

In this paper, a new feature extraction method based on human tissue density patterns, named Analysis of Human Tissue Densities (AHTD), is proposed. AHTD is based on radiological densities of human tissues in Hounsfield Units (HU) following a dedicated approach for medical images. This approach tends to increase the quality of the classification results compared to the current extraction feature methods used. The proposed method is compared against GLCM, IM, SM, ZM, EFF, TF and SCM in pattern recognition experiments using two dierent image databases.

The efficiency of the proposed feature extraction method was evaluated using two CT datasets: one related to brain diseases and the other to lung diseases. Both datasets presented non-regular patterns, uneven illumination and dierent structural characteristics. These distinct CT image datasets were chosen because of their importance to global health. The World Health Organization (WHO) estimates that 300 million people have asthma and this disease causes about 250 thousand deaths per year worldwide [26]; also WHO reported that the number of deaths from chronic obstructive pulmonary disease (COPD) increased by $30 \%$ in 2015, and that this disease will be the third major cause of death worldwide in 2030 [27]. While for brain diseases, WHO estimated that 17.5 million people died of cardiovascular disease in 2012, which is $31 \%$ of all global deaths. Of these deaths, 6.7 million were estimated to be due to brain stroke [28]. In 2012, heart disease and stroke were among the three top causes of premature mortality in the world [29]. Hence, good results obtained with the proposed method would be a potential to improve medical diagnoses and assist in making more accurate and ecient decisions for these two major health problems.

\section{Analysis of Human Tissue Densities}

The concept behind the analysis of human tissue densities using the Hounsfield Units (HU) obtained from CT scanners was proposed by Rebouças Filho et al. [30]. In this study, the authors proposed a decision tree based on the analysis of the neighborhood density to find the best position to initialize an active contour model, and also to decide whether objects of interest are inside or outside the lung under segmentation. Rebouças Filho et al. [31] applied the same concept to achieve the same segmentation task by means of an Artificial Neural 


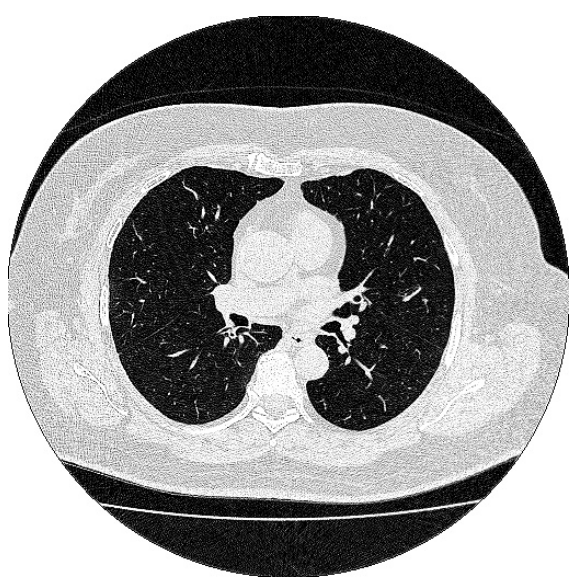

(a)

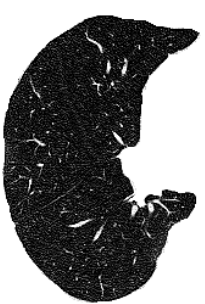

(d)

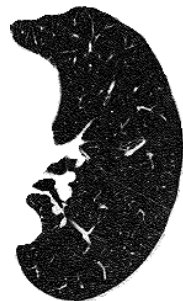

(e)

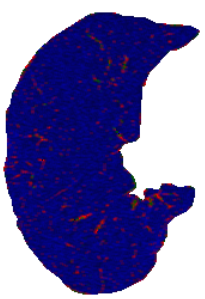

(j)

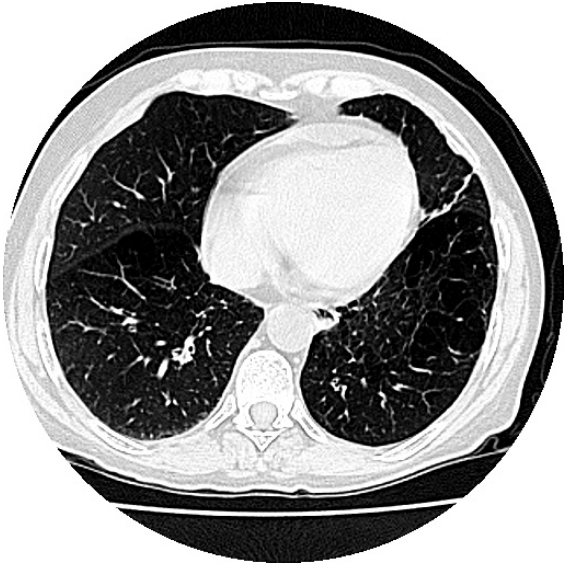

(b)

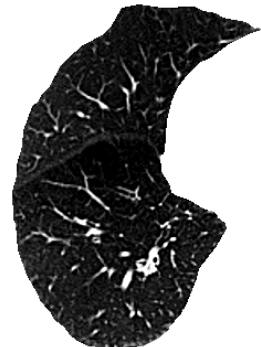

(f)

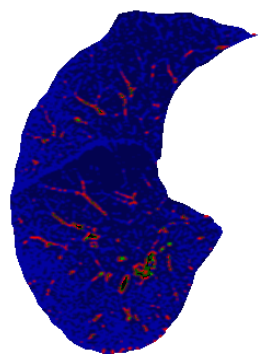

(1)

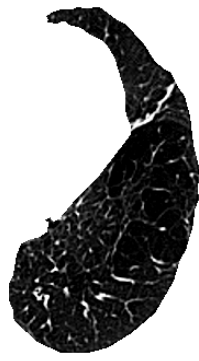

(g)

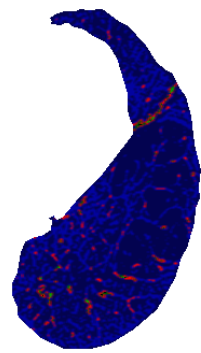

(m)

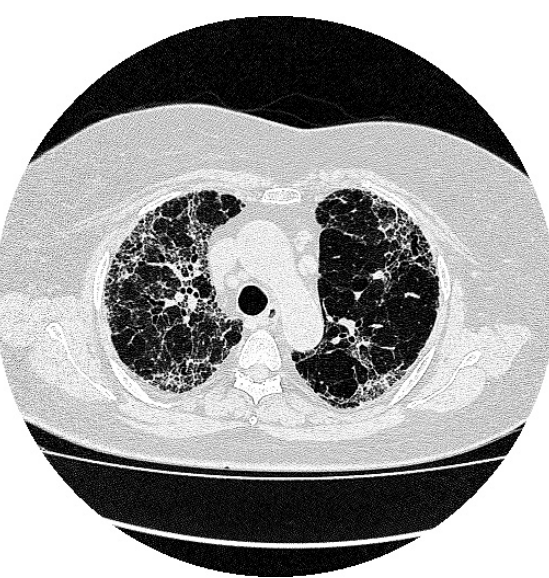

(c)

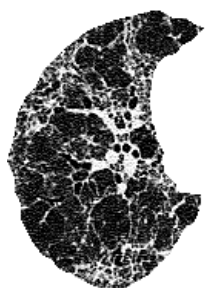

(h)

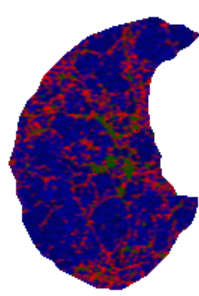

(n)

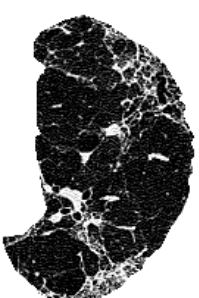

(i)

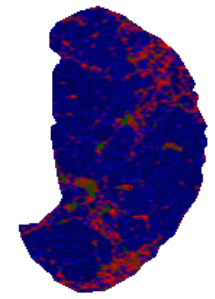

(o)

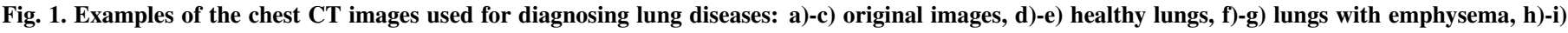
lungs with fibrosis, and j)-o) map of AHTD lung features.

Network. The same concept was also applied to segment the airways [32] and blood vessels [33] inside the lungs in CT images. Here, the same concept was used in the development of a new method to extract features from medical images based on the analysis of human tissue densities in HU, which are particularly interesting for the identification and classification of diseases.

The proposed feature extraction method uses the percentages $P_{i}$ of healthy human tissues based on their density analysis in $\mathrm{HU}$, according to:

$$
P_{i}=\frac{f\left(v_{i}\right)}{\sum_{j=0}^{N-1} f\left(v_{i}\right)},
$$

where $N$ is the number of the tissues under analysis.

Considering a region to be analyzed previously segmented, the function that determines the number of points with densities presented in each class $v_{i}$ is defined as:

$$
f\left(v_{i}\right)=\sum_{x=0}^{W} \sum_{y=0}^{H} R(x, y)
$$

where $W$ and $H$ are the dimensions of the image, width and height respectively, and $R(x, y)$ is given by:

$$
R(x, y)=\left\{\begin{array}{l}
1, \lim _{\text {inf }}\left(v_{i}\right)<T(x, y)<\lim _{\text {sup }}\left(v_{i}\right), \\
0, \text { otherwise }
\end{array}\right.
$$

where $\lim _{\text {inf }}\left(v_{i}\right)$ and $\lim _{\text {sup }}\left(v_{i}\right)$ are the lower and upper limits of the density range in $\mathrm{HU}$ for the class $v_{i}$, and $T(x, y)$ denotes each pixel of the image under analysis.

The proposed feature extraction method was applied to both $\mathrm{CT}$ datasets (lung and brain) using five density classes $\left(v_{i}\right)$ (with $i$ varying from 0 to 4 ) classes. The following classes were used for the lung CT dataset [30]: hyper-inflated air (1000 to 950 $\mathrm{HU})$, normally inflated air (950 to $500 \mathrm{HU}$ ), low inflated air 


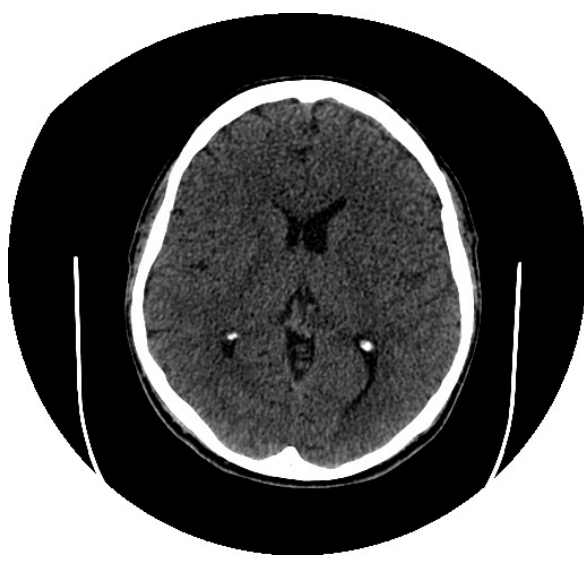

(a)

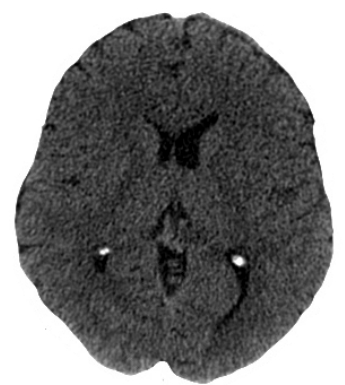

(d)

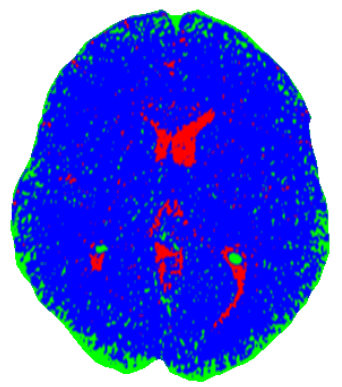

(g)

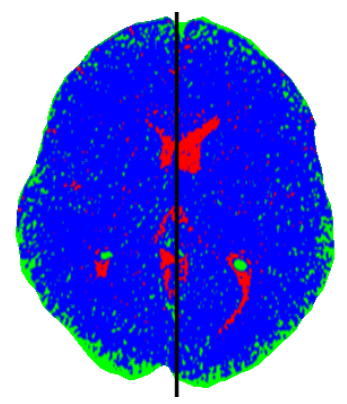

(j)

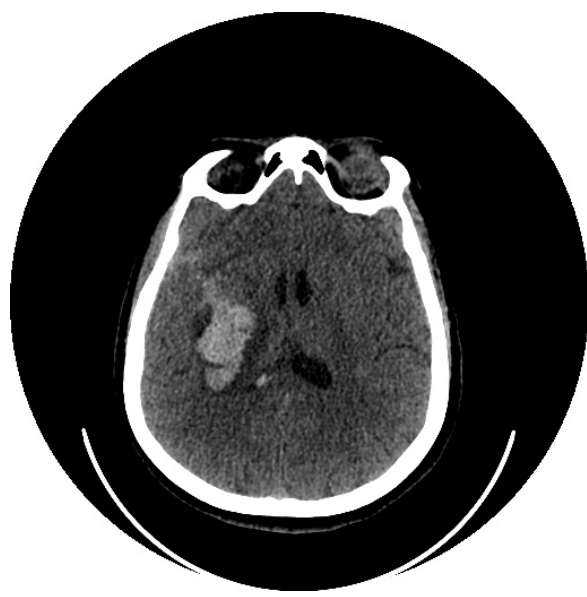

(b)

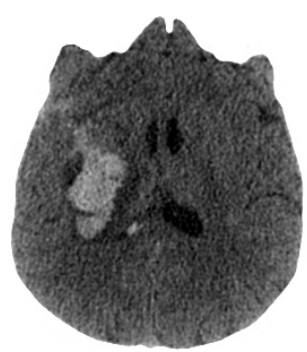

(e)

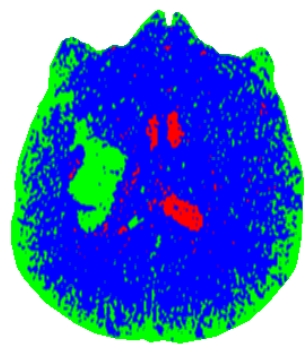

(h)

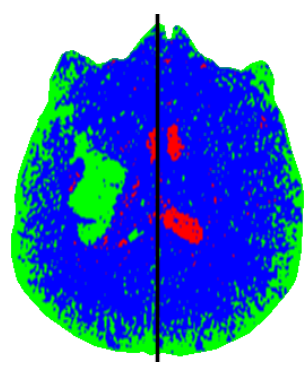

(k)

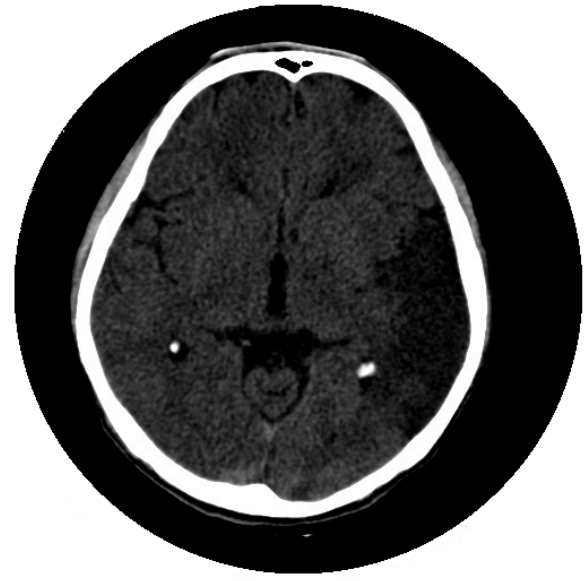

(c)

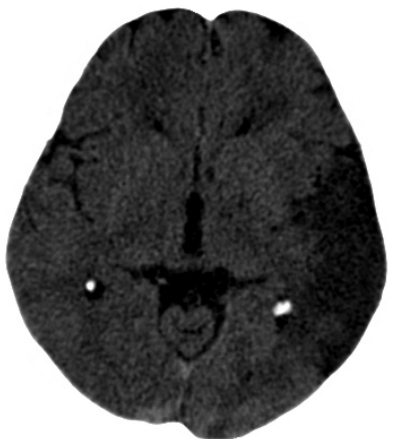

(f)

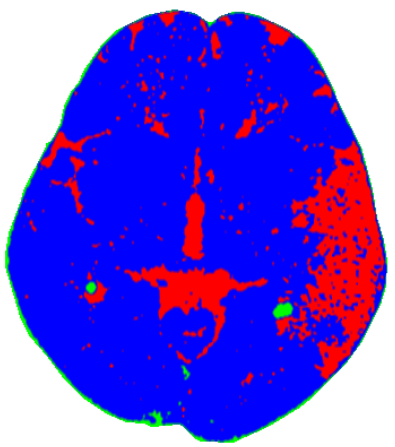

(i)

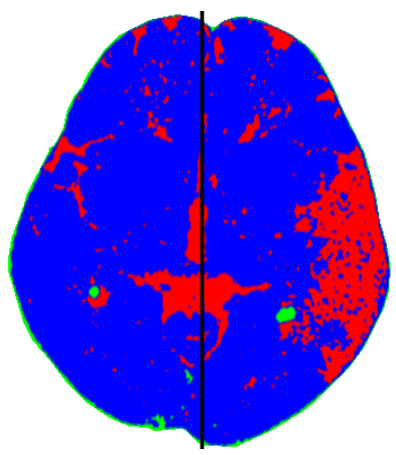

(1)

Fig. 2. Examples of the CT brain images used for diagnosing brain diseases: a)-c) original images, d) healthy brain, e) brain with hemorrhagic stroke, f) brain with ischemic stroke, g)-i) maps of AHTD brain features, and j)-l) brains divided into two regions of interest (ROIs). 
defined as [34]: blood (56 to $76 \mathrm{HU}$ ), bone (200 to $2000 \mathrm{HU}$ ), brain spinal fluid ( -5 to $5 \mathrm{HU}$ ), brain white matter (23 to 34 $\mathrm{HU}$ ) and brain gray matter ( 32 to $41 \mathrm{HU}$ ).

To introduce the features extracted by the proposed method, the third row in the Figs. 1 and 2 show the lung and brain AHTD distribution maps, respectively. The AHTD maps were built in $R G B$ according to:

$$
A H T D_{M A P}=\left\{\begin{array}{l}
R(x, y)=P_{2}, \\
G(x, y)=\frac{P_{3}+2 \times P_{4}}{3}, \\
B(x, y)=\frac{P_{0}+2 \times P_{1}}{3},
\end{array}\right.
$$

where $P_{i}$ is defined by Eq. 1 .

In order to classify the brain diseases, the brain CT image was divided into two regions of interest (ROIs) and the attributes, i.e., the features, were extracted from both ROIs, as shown in the fourth row of Fig. 2.

\section{Datasets description}

CT systems used to acquire image datasets have a resolution of $512 \times 512$ pixels with 16 bits, and the tomographic planes are defined on the basis of the axial plane. The CT chest dataset was obtained from Walter Cantidio University Hospital, Fortaleza, Brazil; they had been successfully used in previous studies [30, 31, 34]. This dataset consists of 12 images of healthy volunteers and 24 patients: 12 with fibrosis and 12 with pulmonary emphysema. Given that each image presents two lungs, there were 24 examples of each class (healthy volunteers, patients with fibrosis and patients with emphysema). The first row of Fig. 1 shows an example image for each class from the dataset. The second row of Fig. 1 shows examples of the lungs under study, which were segmented from the original images using the method proposed in [35]. The grayscale lung images were obtained from the original chest $\mathrm{CT}$ images by using a window with $600 \mathrm{HU}$ of width and centered at $1000 \mathrm{HU}$ [36]. The brain dataset was obtained in partnership with the Hearth Hospital in Fortaleza, Cear, Brazil. This dataset contains 100 images of healthy volunteers and 200 patients: 100 hemorrhagic and 100 ischemic stroke images. The first row of Fig. 2 shows an example image for each class included in this dataset. The second row of Fig. 2 shows examples of the brain CT images under study, which were segmented from the original images using the method proposed in [35, 37]. The grayscale brain images were obtained from the original brain CT images by using a window with $100 \mathrm{HU}$ of width and centered at $50 \mathrm{HU}$ [38].

\section{Results}

In this section, the results of each feature extraction method from the two experimental datasets are presented and discussed in terms of computational cost and classification performance. The tests were performed using a Macbook Pro with an Intel Core i5 $2.4 \mathrm{GHz}, 8 \mathrm{~GB}$ of RAM, and running MAC OS X El Capitan 10.11.2. The proposed feature extraction method was compared against GLCM [39], SM [9], HM [40], ZM [22], EFF
[23] and TF [24]. All of these methods are invariant to rotation, including the isotropic GLCM feature space obtained by computing the correlation, contrast, homogeneity and energy texture descriptors in 4 directions.

The pattern recognition experiments were performed using machine learning algorithms with different settings to obtain the overall classification performance for each feature set built. The classifiers used were: Bayesian [41], Optimum-Path Forest (OPF) with Euclidean distance [42, 43], k-Nearest Neighbor $(\mathrm{kNN})$ with $\mathrm{k}=1$ [44], and Support Vector Machine configured with the radial basis function kernel (SVM) and automatic setup [45]. The classification accuracy was estimated using the 10fold cross-validation method.

The OpenCV 2.4 library was used for all feature extraction methods, except for the new AHTD based method. Figure 3 shows the results of the classification methods in terms of accuracy, f-score, training and test times for the lung and brain CT image datasets. Figure 4 and shows the extraction time required by each feature extraction method under comparison for lung and brain $\mathrm{CT}$ images, respectively.

Figure 3 shows that some feature extraction methods get better results with specific classifiers, such as HM or GLCM when applied together with OPF or SVM in the classification of lung diseases, and SM applied with OPF in the classification of brain diseases. However, it is noteworthy that the proposed AHTD method obtained the best accuracy with Bayes, KNN and OPF for lung disease classification, where the latter has the highest value obtained among all results. When applied on stroke classification, the AHTD method shows the best accuracy with the SVM classifier, and it has the second best result when applied with Bayes, kNN and OPF. For a further analyze, Tables 1 and 2 show the ranking for each feature extractor, classifying each in descending order of accuracy, indicating the best classifier for each feature extractor in each application.

Figure 3 shows that the AHTD based feature extraction method had classification accuracy rates above $95 \%$ for both datasets and with all classifiers; it had the highest average classification accuracy in both cases (for the lungs dataset with the OPF, and for the brain dataset with SVM); it led to classification accuracies in the two highest ones for all classifiers in two application cases, and, finally, the training and testing times were similar to those obtained by the other methods.

Considering the highest accuracy value obtained by each feature extraction method in the lung disease classification shown in Table 1, the AHTD based method had higher values: $0.34 \%$ more than GLCM, $0.86 \%$ more than SCM, $1.48 \%$ more than SM and 9.74\% more than HM, which are the top five results. A similar analysis for the brain disease classification is shown in Table 2 where the AHTD based method also had higher values: $0.16 \%$ more than GLCM, $1.77 \%$ more than $\mathrm{ZM}, 2.54 \%$ more than $\mathrm{TF}$ and $3.26 \%$ more than SM, which are the top five results.

Considering the average of all the results for the lung disease classification, the AHTD based method achieved the best results; the AHTD was 0.72 higher than SCM and $1.77 \%$ higher than GLCM, which are the three top average accuracies. In a similar analysis for the brain disease classification, the AHTD 


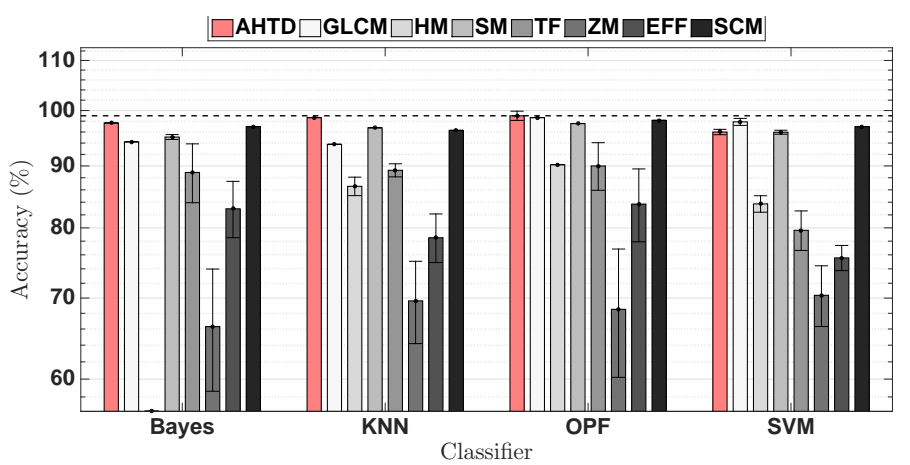

(a)

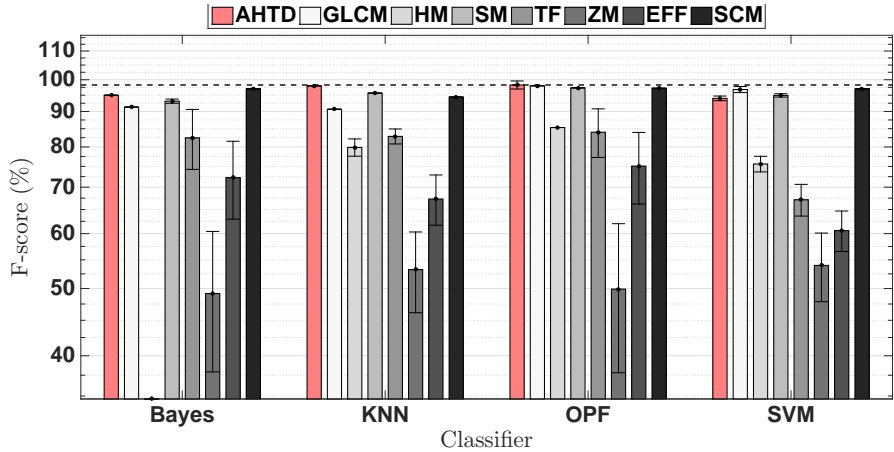

(c)

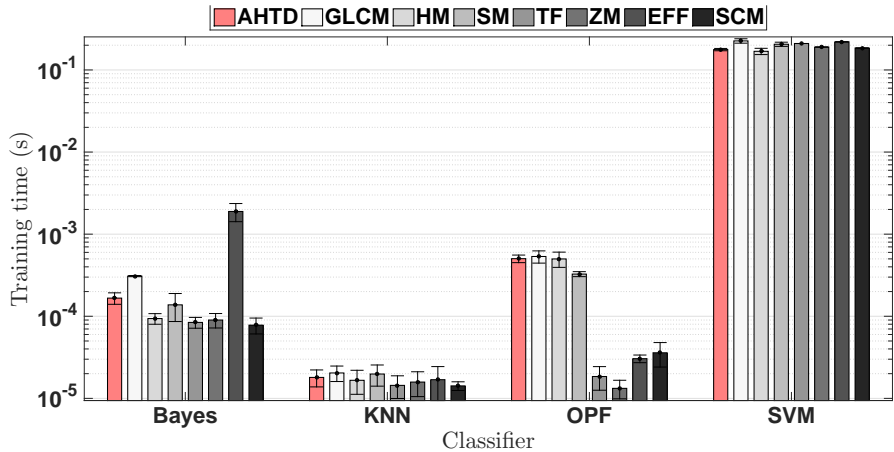

(e)

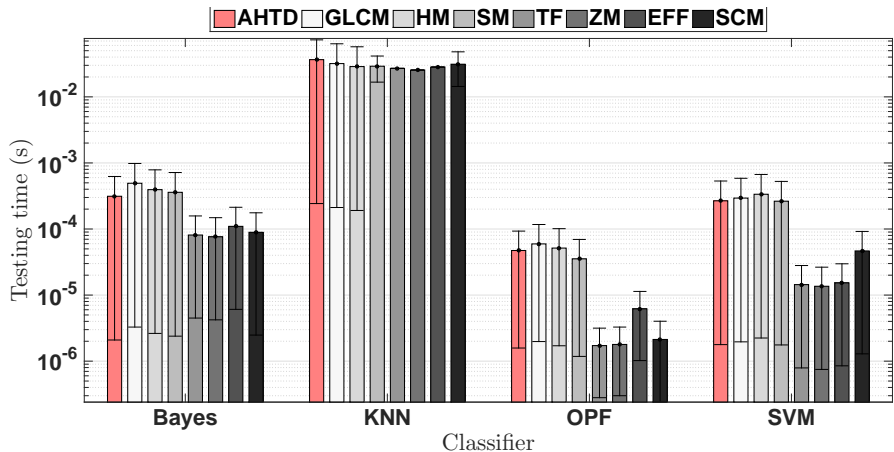

(g)

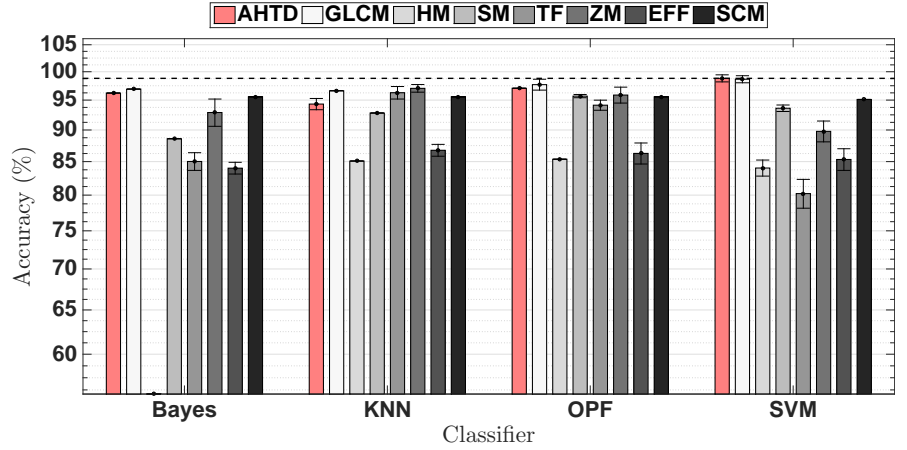

(b)

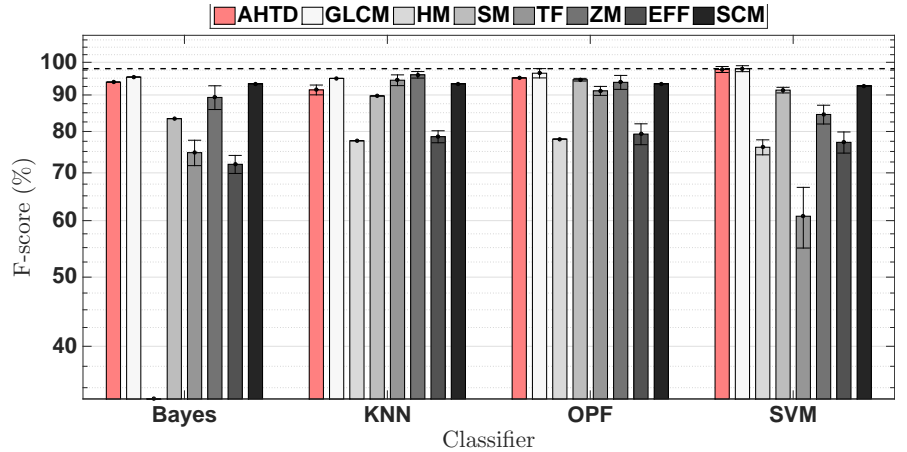

(d)

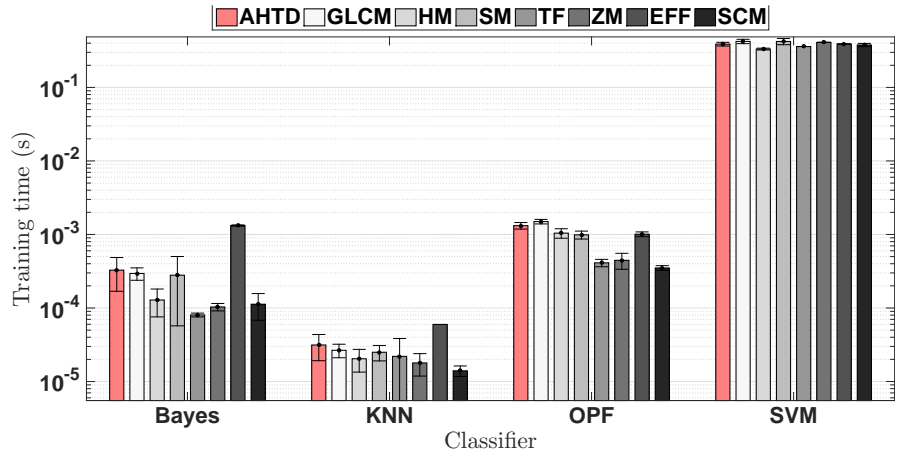

(f)

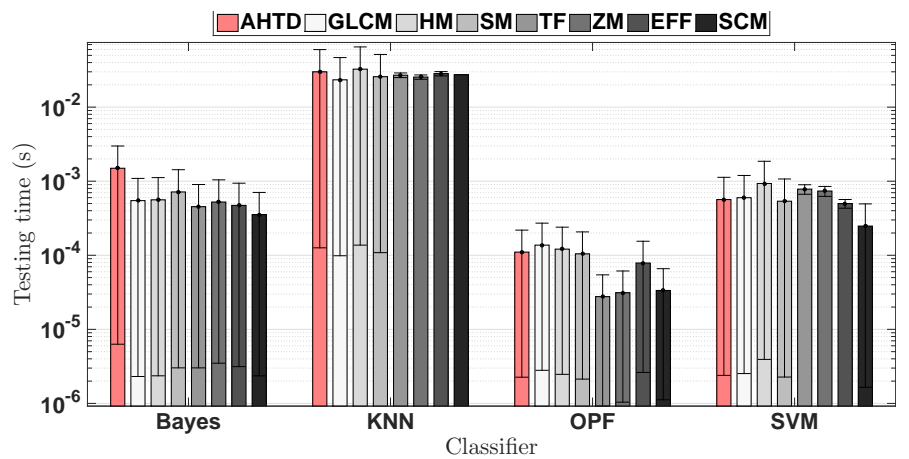

(h)

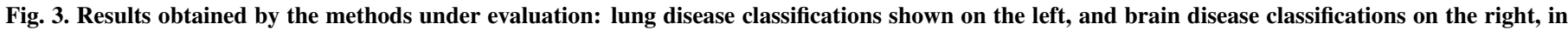
terms of: a) and b) classification accuracy, c) and d) f-score, e) and f) training time, and g) and h) testing time.

based method again shows it had a value $2.71 \%$ higher than ZM and 3.26\% higher than SM, but $0.89 \%$ lower than GLCM.

The AHTD, SM, HM and SCM methods, respectively, are considerably faster than the TF, ZM and EFF methods, respec- tively. The times of each sample analyzed are shown in Figures 4 and 5 for the classification of lung and brain diseases respectively. For a further analysis, Table 3 presents a ranking of the extractors under evaluation considering the shortest ex- 


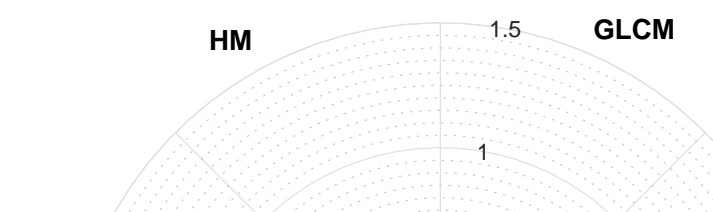

SM

ZM

HM

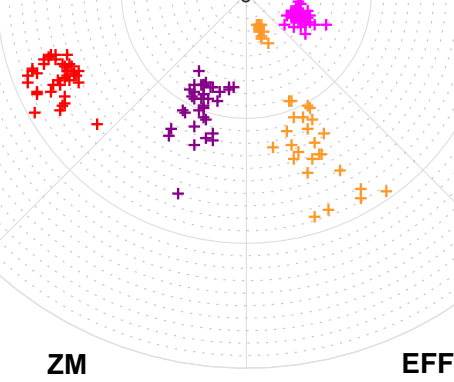

(a)

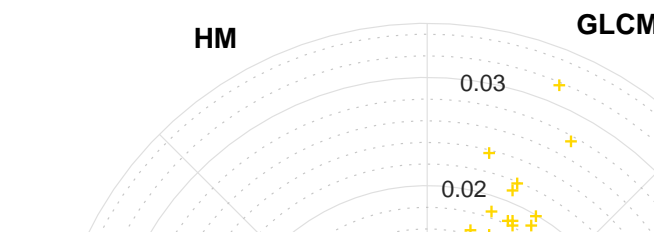

SM

TF

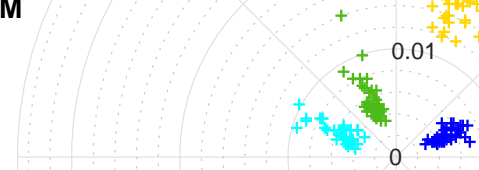

ZM

EFF

(b)

Fig. 4. Time plot of the methods evaluated in the lung disease classification in CT images of the chest: a) time of $0-1.5 \mathrm{~s}$ and b) with zoom, (a) time of 0-0.032s.

traction time to establish the rank order. The order considering the time is the same for the two applications, in which only the AHTD, SM and HM methods have values close to 5 milliseconds, demonstrating that the proposed method also has the best rates in terms of extraction time.

\subsection{Overall results}

Figures 4(b) and 5(b) show the main advantage of the proposed method in the detection and classification of diseases in medical images: the lowest extraction time. In the lung CT image dataset, the proposed method had the lowest extraction time that was equal to $1.3 \mathrm{~ms}$, and 3.52 times faster than GLCM and

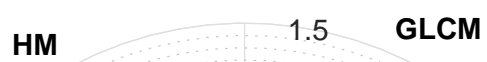

1

SM

0.5

AHTD

TF

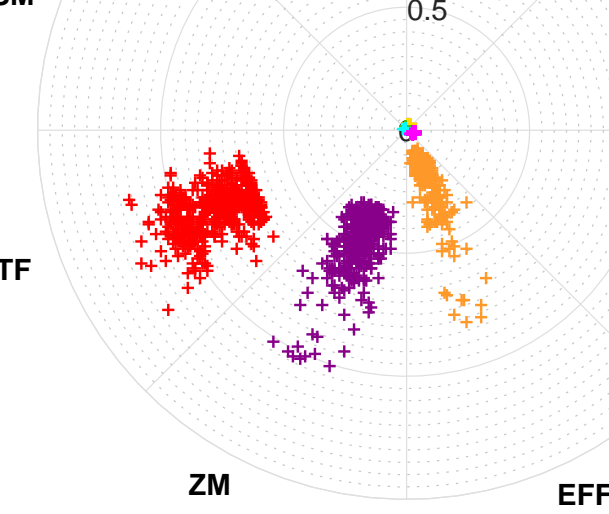

(a)

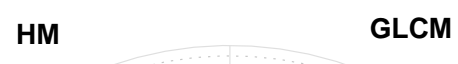

0.03

SM

TF

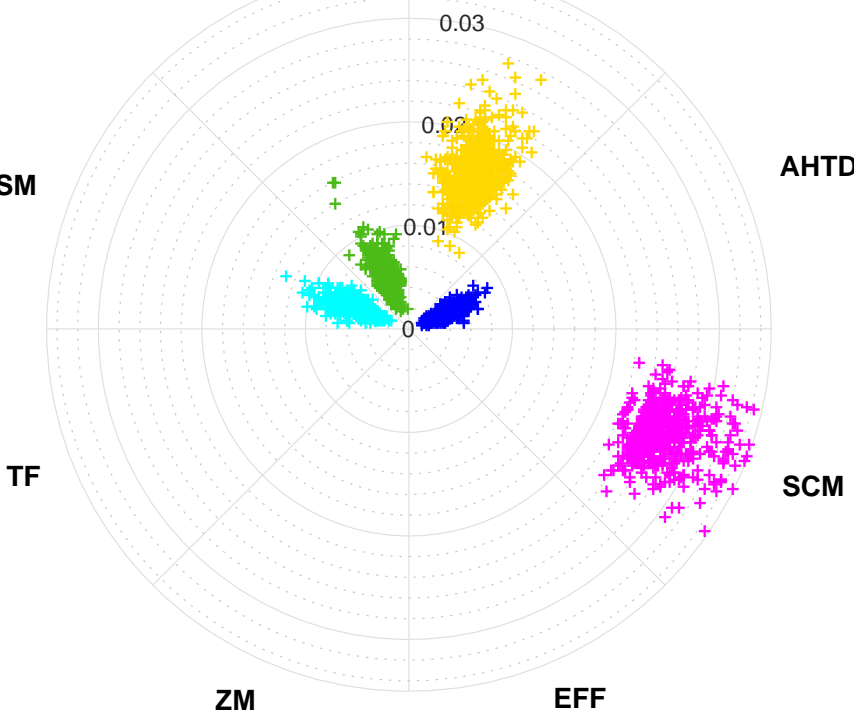

(b)

Fig. 5. Time plot of the methods evaluated in the stroke classification in CT images of the brain: a) time of $0-1.5 \mathrm{~s}$ and b) with zoom, (a) time of 0-0.032s.

1.16 faster than HM and SM. In the brain image dataset, the AHTD based method had an extraction time of $1.4 \mathrm{~ms}$, which was 4.34 times faster than GLCM, 1.39 times faster than HM and 1.44 times faster than SM.

Summarizing the findings, the two best extraction feature methods in terms of the classification accuracy were AHTD and GLCM, which can be confirmed by the F-score values obtained. However, the new AHTD based method required less than 30\% of the time required by GLCM in the extraction process, thus showing the power of the proposed extraction method (AHTD) to classify diseases using medical images. 
Table 1. Statistical results obtained by each feature extractor under evaluation for lung disease classification showing the best classifier, ranked by the maximum (max) accuracy obtained by the feature extractor and machine learning classifier combination. The average (ave) and minimum (min) accuracy also are presented for each feature extractor.

\begin{tabular}{|c|c|c|c|}
\hline Ranking & Feature & Statistic & Accuracy (\%) \\
\hline \multirow{3}{*}{$1^{o}$} & \multirow{3}{*}{ AHTD } & Max & 99.01 \\
\hline & & Ave & 97.83 \\
\hline & & Min & 96.00 \\
\hline \multirow{3}{*}{$2^{o}$} & \multirow{3}{*}{ GLCM } & Max & 98.67 \\
\hline & & Ave & 96.13 \\
\hline & & Min & 93.78 \\
\hline \multirow{3}{*}{$3^{o}$} & \multirow{3}{*}{ SCM } & Max & 98.15 \\
\hline & & Ave & 97.11 \\
\hline & & Min & 96.30 \\
\hline \multirow{3}{*}{$4^{o}$} & \multirow{3}{*}{ SM } & Max & 97.56 \\
\hline & & Ave & 96.34 \\
\hline & & Min & 95.09 \\
\hline \multirow{3}{*}{$5^{o}$} & \multirow{3}{*}{ HM } & Max & 90.22 \\
\hline & & Ave & 79.24 \\
\hline & & Min & 56.44 \\
\hline \multirow{3}{*}{$6^{o}$} & \multirow{3}{*}{$\mathrm{TF}$} & Max & 90.00 \\
\hline & & Ave & 86.94 \\
\hline & & Min & 79.63 \\
\hline \multirow{3}{*}{$7^{\circ}$} & \multirow{3}{*}{$\mathrm{EFF}$} & Max & 83.70 \\
\hline & & Ave & 80.18 \\
\hline & & Min & 75.56 \\
\hline \multirow{3}{*}{$8^{o}$} & \multirow{3}{*}{$\mathrm{ZM}$} & Max & 70.37 \\
\hline & & Ave & 68.70 \\
\hline & & Min & 66.30 \\
\hline
\end{tabular}

\section{Conclusions}

In this work, a novel method for feature extraction from medical images was proposed based on radiological densities of human tissues. The proposed method was compared against the Gray Level Co-occurrence Matrix, Hu's moments, Statistical moments, Zernike's moments, Elliptic Fourier features, Tamura's features and the Statistical Co-occurrence Matrix. For these analyzes, four machine learning classifiers was applied to each feature extractor for the two image CT datasets used: one to classify lung diseases in CT images of the thorax and the other to classify stroke in CT images of the brain.

The proposed AHTD method presented a faster extraction of the required attributes, as well as obtaining the best accuracy and f-score indices in the two datasets evaluated. The attributes from the lung images were extracted in 5.2 milliseconds and obtained an accuracy of $99.01 \%$ for the detection and classification of lung diseases, while the attributes from the brain images were extracted in 3.8 milliseconds and obtained an accuracy of $98.81 \%$ in the detection and classification of stroke. These achievements show that the proposed method can be used in the classification of diseases in medical images, and can be used as an alternative method for real-time applications due to its fast extraction time of suitable attributes.

Having demonstrated the potential of the proposed feature extraction method, future works will be related to the identifi-
Table 2. Statistical results obtained by each feature extractor under evaluation for brain stroke classification showing the best classifier, ranked by the maximum accuracy obtained by the feature extractor and machine learning classifier combination. The average (ave) and minimum (min) accuracy also are presented for each feature extractor.

\begin{tabular}{|c|c|c|c|}
\hline Ranking & Feature & Statistic & Accuracy $(\%)$ \\
\hline \multirow{3}{*}{$1^{o}$} & \multirow{3}{*}{ AHTD } & Max & 98.81 \\
\hline & & Ave & 96.60 \\
\hline & & Min & 94.32 \\
\hline \multirow{3}{*}{$2^{o}$} & \multirow{3}{*}{ GLCM } & Max & 98.65 \\
\hline & & Ave & 97.46 \\
\hline & & Min & 96.62 \\
\hline \multirow{3}{*}{$3^{o}$} & \multirow{3}{*}{ ZM } & Max & 97.04 \\
\hline & & Ave & 93.89 \\
\hline & & Min & 89.78 \\
\hline \multirow{3}{*}{$4^{o}$} & \multirow{3}{*}{$\mathrm{TF}$} & Max & 96.27 \\
\hline & & Ave & 88.91 \\
\hline & & Min & 80.22 \\
\hline \multirow{3}{*}{$5^{\circ}$} & \multirow{3}{*}{ SM } & Max & 95.69 \\
\hline & & Ave & 92.67 \\
\hline & & Min & 88.59 \\
\hline \multirow{3}{*}{$6^{\circ}$} & \multirow{3}{*}{ SCM } & Max & 95.56 \\
\hline & & Ave & 95.44 \\
\hline & & Min & 95.11 \\
\hline \multirow{3}{*}{$7^{o}$} & \multirow{3}{*}{ EFF } & Max & 86.76 \\
\hline & & Ave & 85.59 \\
\hline & & Min & 84.00 \\
\hline \multirow{3}{*}{$8^{o}$} & \multirow{3}{*}{$\mathrm{HM}$} & Max & 85.37 \\
\hline & & Ave & 77.58 \\
\hline & & Min & 55.84 \\
\hline
\end{tabular}

Table 3. Brain and Lung time extraction rankings.

\begin{tabular}{c|c|c|c}
\multicolumn{4}{c}{ Table 3. Brain and Lung time extraction rankings. } \\
Ranking & Feature & $\begin{array}{c}\text { Lung } \\
\text { (seconds) }\end{array}$ & $\begin{array}{c}\text { Brain } \\
\text { (seconds) }\end{array}$ \\
\hline $1^{o}$ & AHTD & $0.0052 \pm 0.0011$ & $0.0038 \pm 0.0011$ \\
$2^{o}$ & SM & $0.0057 \pm 0.0017$ & $0.0055 \pm 0.0016$ \\
$3^{o}$ & HM & $0.0058 \pm 0.0021$ & $0.0053 \pm 0.0017$ \\
$4^{o}$ & GLCM & $0.0167 \pm 0.0042$ & $0.0165 \pm 0.0028$ \\
$5^{o}$ & SCM & $0.0265 \pm 0.0026$ & $0.0271 \pm 0.0029$ \\
$6^{o}$ & ZM & $0.4862 \pm 0.1009$ & $0.4804 \pm 0.1242$ \\
$7^{o}$ & EFF & $0.5229 \pm 0.2755$ & $0.5394 \pm 0.1104$ \\
$8^{o}$ & TF & $0.8346 \pm 0.0641$ & $0.8374 \pm 0.1285$ \\
\hline \multicolumn{3}{|c}{}
\end{tabular}

cation and classification of other diseases in lung and brain CT images as well as in other types of medical images. The identification and classification of structures and organs in the human body are also goals for future works.

\section{Acknowledgements}

Pedro P. Rebouças Filho acknowledges the sponsorship from the Federal Institute of Education, Science and Technology of Ceará, in Brazil, via Grants PROINFRA-IFCE/2013 and PROAPP-IFCE/2014. He also acknowledges the sponsorship from the Brazilian National Council for Research and Development (CNPq) through grants 232644/2014-4. 
Authors gratefully acknowledge the funding of Project NORTE-01-0145-FEDER-000022 - SciTech - Science and Technology for Competitive and Sustainable Industries, cofinanced by Programa "Operacional Regional do Norte (NORTE2020)", through "Fundo Europeu de Desenvolvimento Regional (FEDER)".

Victor Hugo C. de Albuquerque acknowledges the sponsorship from the Brazilian National Council for Research and Development (CNPq) via Grants 470501/2013-8 and 301928/2014-2.

\section{References}

[1] S. F. Eskildsen, P. Coup, V. S. Fonov, J. C. Pruessner, D. L. Collins, Structural imaging biomarkers of alzheimer's disease: predicting disease progression, Neurobiology of Aging 36, Supplement 1 (2015) S23 - S31.

[2] T. Upadhaya, Y. Morvan, E. Stindel, P.-J. Le Reste, M. Hatt, Prognostic value of multimodal mri tumor features in glioblastoma multiforme using textural features analysis, in: Biomedical Imaging (ISBI), 2015 IEEE 12th International Symposium on, 2015, pp. 50-54.

[3] E. C. Neto, P. C. Cortez, T. S. Cavalcante, V. E. Rodrigues, P. P. R. Filho, M. A. Holanda, 3D lung fissure segmentation in TC images based in textures, IEEE Latin America Transactions 14 (1) (2016) 254-258.

[4] M. Abedini, N. Codella, J. Connell, R. Garnavi, M. Merler, S. Pankanti, J. Smith, T. Syeda-Mahmood, A generalized framework for medical image classification and recognition, IBM Journal of Research and Development $59(2 / 3)(2015) 1: 1-1: 18$.

[5] G. L. B. Ramalho, P. P. Rebouças Filho, F. N. S. de Medeiros, P. C. Cortez, Lung disease detection using feature extraction and extreme learning machine, Revista Brasileira de Engenharia Biomdica 30 (2014) 363-376.

[6] E. J. Holmes, A. C. Forrest-Hay, R. R. Misra, Interpretation of emergency head CT: a practical handbook, Cambridge University Press, 2008.

[7] G. N. Hounsfield, Computerized transverse axial scanning (tomography): Part 1. description of system, The British journal of radiology 46 (552) (1973) 1016-1022.

[8] T. P. Naidich, M. Castillo, S. Cha, J. G. Smirniotopoulos, Imaging of the brain: expert radiology series, Elsevier Health Sciences, 2012.

[9] M. Nixon, M. S. Nixon, A. S. Aguado, Feature extraction \& image processing for computer vision, Academic Press, 2012.

[10] A. Paul, N. Bhattacharya, A. Chowdhury, Digit recognition from pressure sensor data using euler number and central moments, in: Communications, Devices and Intelligent Systems (CODIS), 2012 International Conference on, 2012, pp. 93-96.

[11] T. Czachorski, S. Kozielski, U. Stanczyk, Man-Machine Interactions 2, Advances in Intelligent and Soft Computing, Springer Berlin Heidelberg, 2011.

[12] M.-K. Hu, Visual pattern recognition by moment invariants, Information Theory, IRE Transactions on 8 (2) (1962) 179-187.

[13] Z. Huang, J. Leng, Analysis of Hu's Moment Invariants on Image Scaling and Rotation, in: Computer Engineering and Technology (ICCET), 2010 2nd International Conference on, Vol. 7, Changdu, China, 2010, pp. 476480 .

[14] Y. Sun, G. Wen, J. Wang, Weighted spectral features based on local Hu moments for speech emotion recognition, Pattern Recognition Letters 18 (2015) 80-90.

[15] J. Flusser, T. Suk, J. Boldys, B. Zitova, Projection operators and moment invariants to image blurring, Pattern Analysis and Machine Intelligence, IEEE Transactions on 37 (4) (2015) 786-802.

[16] R. Haralick, Statistical and structural approaches to texture, Proceedings of the IEEE 67 (5) (1979) 786-804

[17] P. Li, X. Liu, N. Zhao, Weighted co-occurrence phase histogram for iris recognition, Pattern Recognition Letters 33 (2012) 1000 - 1005.

[18] M. Gupta, D. Bhaskar, R. Bera, S. Biswas, Target detection of ISAR data by principal component transform on co-occurrence matrix, Pattern Recognition Letters 33 (2012) 1682-1688.

[19] S. Beura, B. Majhi, R. Dash, Mammogram classification using two dimensional discrete wavelet transform and gray-level co-occurrence matrix for detection of breast cancer, Neurocomputing 154 (0) (2015) 1 14.
[20] S. Chu, L. Hong, C. Liu, J. Chen, A new regional shape index for classification of high resolution remote sensing images, in: Earth Observation and Remote Sensing Applications (EORSA), 2014 3rd International Workshop on, 2014, pp. 156-160.

[21] M. Alves, E. Clua, F. Leta, Evaluation of surface roughness standards applying haralick parameters and artificial neural networks, in: Systems, Signals and Image Processing (IWSSIP), 2012 19th International Conference on, 2012, pp. 452-455.

[22] A. Khotanzad, Y. H. Hong, Invariant image recognition by zernike moments, IEEE Transactions on pattern analysis and machine intelligence 12 (5) (1990) 489-497.

[23] F. P. Kuhl, C. R. Giardina, Elliptic fourier features of a closed contour, Computer graphics and image processing 18 (3) (1982) 236-258.

[24] H. Tamura, S. Mori, T. Yamawaki, Textural features corresponding to visual perception, IEEE Transactions on Systems, Man, and Cybernetics 8 (6) (1978) 460-473.

[25] G. L. B. Ramalho, D. S. Ferreira, P. P. R. Filho, F. N. S. de Medeiros, Rotation-invariant feature extraction using a structural co-occurrence matrix, Measurement 94 (2016) 406 - 415.

[26] H. Campos, A. Lemos, Asthma and COPD in view of the pulmonologist, Brazilian Journal of Pulmonology 35 (4).

[27] WHO, Global strategy for the diagnosis, management and prevention chronic obstrutive pulmonar disease, Tech. rep., World Health Organization (2014).

URL http://www . who.int/respiratory/copd/causes/en/

[28] WHO, Cardiovascular diseases (cvds), Tech. rep., World Health Organization (2015)

URL http://www.who.int/mediacentre/factsheets/fs317/ en/

[29] S. Mendis, T. Armstrong, D. Bettcher, F. Branca, J. Lauer, C. Mace, V. Poznyak, L. Riley, V. Silva, G. Stevens, et al., Global status report on noncommunicable diseases 2014, Switzerland: World Health Organization (2014) 14-15.

[30] P. P. Rebouças Filho, P. C. Cortez, M. A. Holanda, Active contour modes crisp: new technique for segmentation the lungs in CT images, Revista Brasileira de Engenharia Biomdica 27 (2011) 259-272.

[31] P. P. Rebouças Filho, P. C. Cortez, J. H. S. Felix, T. da Silveira, M. A. Holanda, Adaptive 2D crisp active contour model applied to lung segmentation in CT images of the thorax of healthy volunteers and patients with pulmonary emphysema, Revista Brasileira de Engenharia Biomdica (2013) 363-376.

[32] T. S. Cavalcante, P. C. Cortez, P. P. Rebouças Filho, J. H. S. Felix, A. R. Alexandria, M. A. Holanda, Comparative analysis of segmentation techniques of airways on images of chest computed tomography, in: 17th International Conference on Systems, Signals and Image ProcessingIWSSIP, IWSSIP, Rio de Janeiro, RJ, 2010, pp. 1-4.

[33] A. B. N. Ribeiro, Lung blood vessels segmentation in thoracic ct scans, Master's thesis, Universidade Federal do Ceará, Fortaleza, CE (2013)

[34] P. P. R. Filho, Cortez, P. César, A. C. S. Barros, V. H. C. Albuquerque, J. ao Manuel Ribeiro Silva Tavares, Novel and powerful 3d adaptive crisp active contour method applied in the segmentation of ct lung images, Medical Image Analysis 35 (2017) 503-516.

[35] P. P. R. Filho, Cortez, P. César, A. C. S. Barros, V. H. C. Albuquerque, Novel adaptive balloon active contour method based on internal force for image segmentation - A systematic evaluation on synthetic and real images, Expert Systems with Applications 41 (17) (2014) 7707-7721.

[36] W. R. Webb, W. E. Brant, N. M. Major, Fundamentals of body CT, Elsevier Health Sciences, 2014.

[37] F. D. L. Moreira, M. N. Kleinberg, H. F. Arruda, F. N. C. Freitas, M. M. V. Parente, V. H. C. de Albuquerque, P. P. R. Filho, A novel vickers hardness measurement technique based on adaptive balloon active contour method, Expert Systems with Applications 45 (2016) 294 - 306.

[38] D. M. Yousem, R. I. Grossman, Neuroradiology: the requisites, Elsevier Health Sciences, 2010.

[39] R. Haralick, K. Shanmugam, I. Dinstein, Textural features for image classification, Systems, Man and Cybernetics, IEEE Transactions on SMC3 (6) (1973) 610-621.

[40] M.-K. Hu, Visual pattern recognition by moment invariants, Information Theory, IRE Transactions on 8 (2) (1962) 179-187.

[41] O. Sayadi, M. B. Shamsollahi, A model-based bayesian framework for ECG beat segmentation, Physiological Measurement 30 (3) (2009) 335352. 
[42] J. P. Papa, A. X. Falcão, C. T. N. Suzuki, Supervised pattern classification based on optimum-path forest, International Journal of Imaging Systems and Technology 19 (2) (2009) 120-131.

[43] E. C. Neto, S. L. Gomes, P. P. R. Filho, V. H. C. de Albuquerque, Brazilian vehicle identification using a new embedded plate recognition system, Measurement 70 (2015) $36-46$.

[44] S. L. Gomes, E. d. S. Rebouças, E. C. Neto, J. P. Papa, V. H. C. d. Albuquerque, P. P. Rebouças Filho, J. M. R. S. Tavares, Embedded real-time speed limit sign recognition using image processing and machine learning techniques, Neural Computing and Applications (2016) 1-12.

[45] C.-C. Chang, C.-J. Lin, Libsvm: A library for support vector machines, ACM Trans. Intell. Syst. Technol. 2 (3) (2011) 27:1-27:27. 